\title{
Innovating iTV Browsing: Designing an Affective Search Engine
}

\author{
Calliope Georousi \\ UCL Interaction Centre \\ MPEB 8th floor, University College London, Gower Street, \\ London WC1E 6BT, United Kingdom \\ calliope.georgousi.11@ucl.ac.uk
}

\begin{abstract}
Theory supports the use of emotion and cognition to reach more informed decisions. Whilst, sound technologies are leading on affective search mechanisms, video technologies, such as iTV, have yet to follow. This research addresses iTV content information overload through the design of an affective search methodology, by combining emotion with cognition, to aid users decisions on watched content.
\end{abstract}

A Research through Design approach aimed for innovation, user behaviour support and development. A collaborative thematic analysis then revealed that users' browsing decisions are determined by their transient moods. Participants preferred to search by mood and reason combined. They expressed an interest in playful browsing and self-awareness tools, helping them understand their emotions.

A novel interactive search system was then prototyped, consisting of mood inducing components, building a user archive of mood related video content, yielding personalised real-time recommendations. Moods could be translated and queried through a proposed html 5 mood tag. The resulting search engine supports worded and 'tacit browsing'. In conclusion, more can be known in the $\mathrm{HCl}$ community on adding affect for iTV search and tackling content finding challenges, by the search UI designed.

iTV. Affect. Content overload. RtD. Cultural probes. Co-design workshop. UI prototype. Search engine. Tacit browsing. Mood. Innovation.

\section{INTRODUCTION}

Interactive TV (iTV) is television combined with internet. This adds a layer of 'dialogue' between broadcasted TV and viewers, actively engaging them in making more informed decisions and extending possibilities about the video content they decide to watch. In other words, iTV promotes passive television viewers to proactive users, enhancing their experience, by giving them a new dimension of options.

One of the challenges iTV faces is content finding. According to Lugmayr et al. (2004, in Lino et al. 2011), iTV is bound to face similar challenges to the Web, like complexity and huge information volumes. Video files are normally supplied with insufficient metadata, incommoding browsing: complex navigation, alphabetical categorisation, and long lists of search results to scroll through may be confusing and time consuming. In parallel, the daily increase of content rapidly exacerbates the problem and this is apparent in all platforms reviewed. Consequently, content finding is becoming a Herculean feat for the user to tackle.

Current solutions include Content Strategy Theories and Artificial Intelligence (Al) Technologies, such as content personalisation and localisation, but they do not address affect as a possibility for content finding. Content Strategy theorists, for example, suggest not only to produce less page content, but even to delete previous material (Dalen 2011). Technological solutions (AI) like Google resolved to provide users with personalised search results (Vanderbilt 2013), yet again they fail to introduce affective human mechanisms in the browsing process. All mentioned theories and technologies employ the user's cognition: one must be linguistically aware of the word in search, before the search begins. The user is called to make an decision on what to search to watch.

Research suggests that "people make decisions based upon reason and emotion combined" (Damasio 2005). Although previous work in sound (Stereomood 2008) and image (Getty Images, 1999-2008) have addressed content finding through affect 
(mood), hardly have mood and wordless search mechanisms been considered for iTV browsing. Therefore, the present study primarily explores the concept of iTV mood browsing and how its integration with cognition may help the user's decision making process and enrich their iTV experience. More specifically, it is structured along the following three research questions:

- "Can we use reason together with emotion to browse for iTV content?"

- "Can we browse for iTV content without using words?"

- "How can we, in the $\mathrm{HCl}$ field, design an interaction (system /UI), to support people's iTV browsing decisions?"

The study also explores the concept of wordless (tacit) forms of browsing: "cases where the user's intention is clear, but where the intention may still be identified in linguistic terms" (Boehner et al. 2007).

This paper is structured as follows. Research is conducted in academia, current iTV commerce platforms, related technologies and User Interface Design (UI). A pilot study precedes the main. Cultural Probes and Co-Design methods shed light on user behaviour, needs and browsing preferences. Data is then gathered and thematically analysed. The results of this study suggested search prototype leverage the knowledge of iTV browsing behaviours in $\mathrm{HCl}$ community and potentially inform future trends on iTV search engine models.

\section{LITERATURE REVIEW}

\subsection{Affect and cognition}

Current browsing decisions are made entirely by employing users' cognition. Affect is not included, since users currently cannot browse by typing to receive relevant results (Mosco 2004). Related work on people's decision process is found in the field of Affective Interaction, with Damasio's ground breaking work revealing that:

\begin{abstract}
"knowledge and reasoning alone cannot compensate for making advantageous decisions. Emotion aids in the decision making process and that implementation of decisions under certainty or uncertainty engage different neural circuitries."
\end{abstract}

(Damasio 2005, p.336)

Following Damasio's research, this paper sets out to explore whether a system designed to embrace both cognitive and affective user capabilities, can aid the users content finding process and as such minimise the content overload effect in overall.

\subsection{Tacit browsing}

This study is specifically interested in wordless forms of browsing, be they mood related or not. That is, when the users are aware of what they are looking for, yet they can't express it in linguistic terms. They will however readily recognise it, when they see it. For example, the user may not have a linguistic example of the film title they are looking for, but if they see it, they will readily recognise it. This is state of mind is hereby recognised as "Tacit Browsing".

\subsection{Related work in academia}

Related work in the academia in the field of iTV started in 2004 when Zimmerman et al. (2004) designed a TV Show Recommender Engine and Interface. At the same time Bosma (2004) was using biosensors to detect mood. Much later Zhang et al. (2009) utilised affective analysis for efficient movie browsing. Lino et al. (2011) followed by designing "Knowledge TV", an iTV classification platform based on semantic web concept, to present a new layer of search queries and personalised recommendations. Around the same time Martins et al. (2011) wrote "Movie Clouds", a project focusing on user's mood and engagement, supporting serendipity and wordless browsing. A more recent and relevant work comes from the BBC research team (Eggink et al. 2012) who published their pilot study results on a mood classification system for iTV archived content. Lastly, related academic work concludes with a recent Wired magazine article by Vanderbilt (2013), describing the research Google is undergoing, moving from information retrieval, to a search engine that makes connections and retrieves results. This is in essence a "Knowledge Graph" and it is searching for serendipitous links. However, he concluded by questioning the limitations of knowledge graph technology:

"what happens when knowledge graph is not en-
capsulated in structured databases, when it's not
a piece of text" (Vanderbilt 2013).

Most related work asserted that mood can be used as metadata to facilitate large video archives and supported serendipity, fun and quick retrieval for user engagement along with personalised recommendation accuracy. However, they do not assemble components into a coherent UI to combine affect and cognition, they do not explicitly address tacit forms of iTV browsing. Thirdly, they do not appear to follow an anthropocentric based method, to inform their study and designs.

\subsection{Related work in public commerce platforms}

Research focus then shifted to the current state of the iTV in public commerce technology for findings. The research area for iTV browsing reviews covered UK and Irish iTV platforms, namely, BBC iPlayer, BBC Archives, Channel 4oD, iTV, Sky on the Go, Rté. More basic Ul's and features are found, than those proposed in academia. Some iTV systems include personalised recommendations, as shown in 
Finish iTV, yet affect is certainly not addressed, as the users cannot search via inputting their mood in situ (i.e. type joyful to find related video content). It is then understood that this study is exploring an under-researched area. There is scarcity in designing for iTV UI's that address cognitive-affective browsing mechanisms, follow the UCD approach and consider non-textual browsing.

\subsection{Designing for innovation}

It is understood that the interaction sought to be designed here is novel and the problem to be addressed is "wicked" (Rittel \& Webber 1973), because there is no precedent example and it is not known where the study is leading. Due to its innovative nature, it was deemed better that the resulting system be collaboratively designed with the users, to better address their behaviour, lifestyle and needs. For this reason, Research through design (RtD) provided a structured roadmap. Another influencing approach to the design of novel experiences was turning to "technology for inspiration" (Rogers et al. 2002) who looked in neighbouring fields for connections. Likewise, this research lead to music technologies and mood board in search for possible UI design solutions.

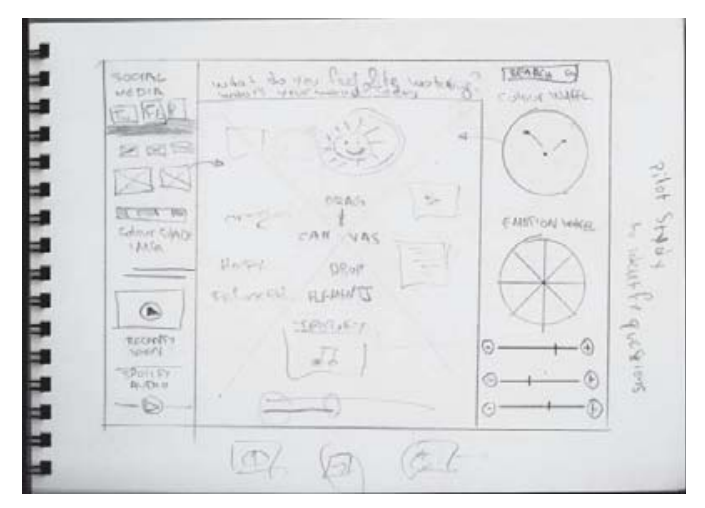

Figure 1: Mood Board UI

\subsection{Design Findings}

\subsubsection{Mood Board UI}

The aim is to aid the user form a collage of mood theme, namely a gestalt of their affective state to search with. Selected research work covers Pulse, a mosaic interface of RSS aggregated feeds; Pinterest, an image and video pin board of bookmarks; mural.ly, a state of the art multimedia mood board; and Moodstream, created by Getty Images, which allows the creation of multimedia playlists to be reused or shared socially. A mood board interface was sketched under the same criteria of practicality and feasibility of implementation, it consists of an empty space with two side columns containing six components.

\subsubsection{Sound technologies}

Sound technologies in the public commercial world are found to be more mood based and matured, than that of iTV. Distinguishable work includes Stereomood, which offers a crowdsourced tag cloud and a mood flip component; Ghostly Discovery's minimalistic and quirky interface which includes a colour wheel with 2 valence sliders; Musicovery, an unconventional quadrant UI, which uses affect mapping to retrieve related music stored in the users device. An additional reason why this study focused on sound technologies for innovation, as Gao and Berthouze (2012) have highlighted, there is a growing need for such technologies. This research on affect and cognition then advocates for a design amalgam, employing users mental model.

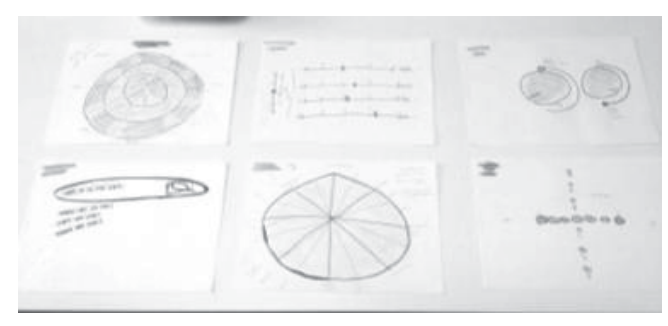

Figure 2: Six Components paper sketched

\subsubsection{Components}

Six mood based components were designed under the criteria for practicality and feasibility of design implementation. A colour wheel provides a lexicon of colour to affect; image recognition illustrates tacit affect; an image upload feature gives the user the creative choice to frame their mood; the mood flip component overturns moods to produce the opposite affect; keyword tag cloud is a collective of mood related words displayed to aid tacit browsing; voice recognition technology detecting words and nuances in a sentence; and finally Sliders define the scale of pleasure or magnitude of felt mood.

\section{METHOD}

\subsection{Main Cultural Probes Method}

Cultural Probes is an 'ethnographic' type of method combining the approach of both Crabtree et al. (2003) and Gaver (2004). Materials used in given tasks, evoked users' thoughts and sparked their imagination (Sanders 2002), igniting a co-operative designer-participant relationship (Lucero 2012). The Main Study was informed by the pilot and appropriate amendments were applied. Task times were also reduced. 


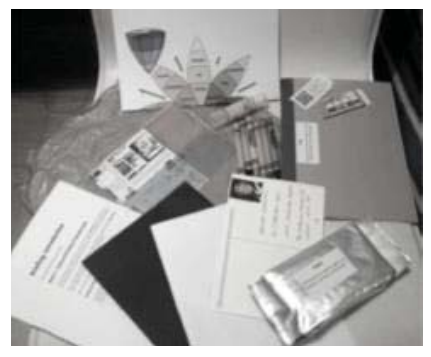

Figure 3: Cultural Probes pack

\subsubsection{Participants}

Seven participants were fully committed, and came up with questions, ideas and stories. All participants returned the Probes. However, most of the participants submitted them after the Participatory Workshop set date, which called for adjustments. Only during the main study, when participants submitted their probes, they were compensated for their participation.

\subsubsection{Materials}

Cultural probe packs (Gaver et al. 2007) included pastel colours, pencils and glue, cards with online links of digital mood board as described below and instructions. The following material was included in the probes pack as described below along with a rationale for their use:

- Mood boards - to create a collage-theme of their mood whilst browsing for iTV videos. An A3 paper size and www.mural.ly private website was provided. An example was set on the website to guide them for the first time.

- A Drawing Experience - to freely sketch their dreams and aspirations under the theme question: "what if a mood search engine existed?"

Participants were provided with 5 A4 print papers with questions labelled on each day.

- A Diary - with instructions asking participants to document their daily browsing stories (Lucero et al. 2012) and express incidents of wordless browsing.

Each day was labelled with a question suggesting a different technology topic, the last two had an open technology topic of their choice.

- A Discrete emotions wheel (Plutchik 2001) - to propel participants mood and aid the researcher during the Data Gathering and Analysis, by giving a lexicon of emotions.

- A single use Kodak Camera (Analogue) - with instructions asking participants to document their personal stories and capture impressions of moments or objects. There are 27 shots in the film, to compliment the diary, mood-board and postcards alike.

- Collage Postcards - with labelled questions about their impressions from the Probes. The postcards already stamped and addressed to the researcher, should be sent back at the end of the week. Written impressions provided the researcher with feedback and the users with a debriefing opportunity.

\subsubsection{Procedure}

Participants were greeted and a Probes pack was given as a present to each participant with the briefing document included in the pack. Briefing session lasted around 30 minutes. Participants were then talked through on how to use the pack. They were then requested to return the probes in a week's time and parted. The researcher emailed private link invitations to the participants and kept within reach for further questions. After the end of the study the pack was allowed to be kept as memorabilia. The participants who could physically meet to return the probes, were audio recorded and notes taken down about the participants' impressions, ideas or thoughts. They were then thanked for their input and departed. The procedure duplicated the pilot study.

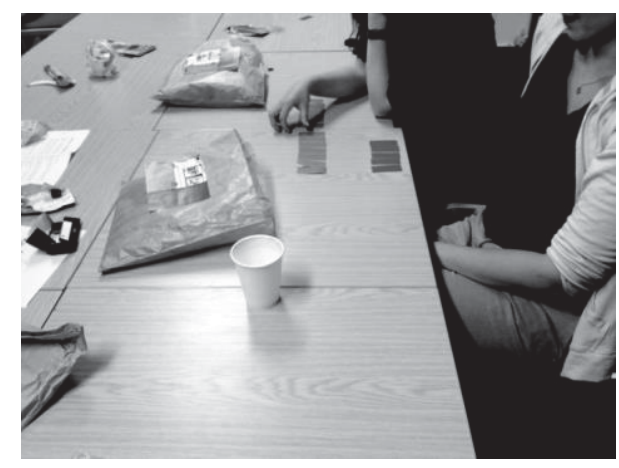

Figure 4: Participants card sorting task

\subsection{Main Co-Design Workshop Method}

Collaborative Design, similar to Lucero (2011) and Sanders (2002), is a workshop characterised by a synergic design process between participants and designers. It was set to inform the design process and evaluate the third research question of this study. It was selected, because it is both research and design-led (Lucero 2011). The Main Study was informed by the pilot; and major changes by clarifying all briefs. Brief wording was improved with the addition of task mood board examples, presentations and samples of components. It also reduced the time estimated for task completion. The same procedure was followed as in the pilot study. However there were some changes noted.

\subsubsection{Participants}

Only three participants appeared to take part. Most of the participants submitted their probes after the Participatory Workshop set date. Upon probes return, participants were compensated for their participation.

\subsubsection{Materials}

Participatory Workshop material consisted of paper drawing material as in the probes. Two packs of small business cards were crafted. An iPad and 
MacBook Pro laptop were provided to users. After the Pilot study, drawing materials were added to the pack. Detailed labelling of each material showed how it could be used, to avoid confusion noted during the pilot study.

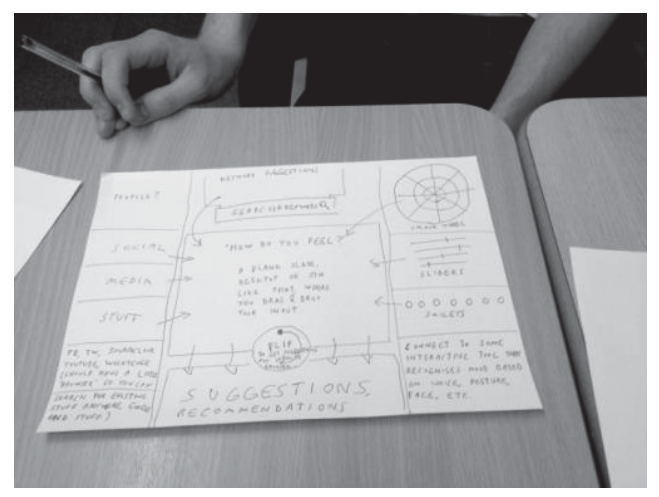

Figure 5: Participant's O UI sketch

\subsubsection{Design and Procedure}

The participatory method, was designed, utilising "Diversifying experience" (Ritter et al. 2012), a model to enhance cognitive flexibility and give users room for creativity outside the realm of "normality." (Horizon - 2013-2014: The Creative Brain: How Insight Works, video recording, 14 March 2013, BBC Two, 43'). The workshop should last no longer than two hours. Namely, the outline of three user tasks was as follows:

Part 1. In the first part, lasting for up to 30 minutes, time was allocated to the return of the probes and a group feedback discussion. The participants were welcomed, then briefed on the tasks. During the main study this task was replaced by a Q\&A session regarding the probes, because the probes were not completed duly. The aim of this part was realised, as participants were fully engaged and had a chance to get acquainted.

Part 2. In the second part, lasting up to one hour, participants were asked to pair up and collaborate on three sets of sub tasks:

- Participants were asked to browse for content by their usual way. They browsed in BBCiPlayer, iTV and Channel 4 on demand. In the pilot study they were asked to browse for the keyword "David Attenborough" and in the main, "Formula One". The keyword was checked to exist prior to the study. - They were then requested to browse for content in an unusual way, by changing one of the steps. Participants browsed for the same iTV content as before, but without using keywords.

- After this, they were provided with a briefing task to evaluate the components and immediately after they were given two minutes resting time. During this time users are requested to get involved in a nondemanding task, of card sorting by colour. For the main study, more blue and red cards were added, since participants were spontaneously grouping together and sorting them in a fraction of time as initially allocated. Resting time was just as important, because it allowed their mind to wander (Baird et al. 2012).

Part 3. In the third and last part, participants were paired and suggested to design an UI of their preference based on the components presented and to create some of their own. This task was set to evaluate the Literature Review findings. The users then presented their sketches to the groups with their rationale, gave feedback and discussed along with the designer for one more iteration. Three UI designs were created during this workshop. Iteration phase integrated all the components. A post- questionnaire followed, engaging the participants in post discussion on the feasibility of the suggested designs. In both pilot and main study, this phase spurred further discussion and brainstorming about apps, future technologies and their use. The users then were escorted to the exit and departed.

\subsubsection{Ethical Considerations}

Participants were informed about the purpose of this study included in the Information Sheet provided at the beginning of the workshop. Their Data anonymity was also ensured under Ethics Form Approval No. Z6364106/2013/05/50. A debriefing session was offered in both studies. Ethical considerations were identical to the pilot.

\section{DATA GATHERING}

The data gathered was then transcribed using the IA writer app and Apple Final Cut Pro X application. Sketches, diaries and created artefacts were digitised. This heterogeneity of material structured the triangulation of the data analysis and its findings, as presented in the following section.

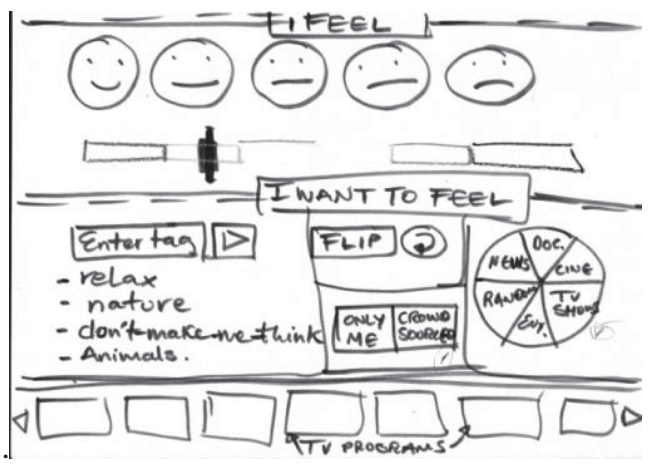

Figure 6: Participant's S UI sketch

\section{ANALYSIS AND FINDINGS}

This section reports on findings from Main Cultural Probes and Participatory Co- Design workshop. The approach used in this research was an inductive 
Thematic Analysis (Braun \& Clarke 2006). The findings were then evaluated through a Research through Design Model (RtD) (Zimmerman et al. 2007). Three themes emerge from the dataset: user behaviour, user concerns, and controversies.

\subsection{User Behaviour Theme Findings}

The user behaviour theme informed this study about the participant's culture in relation to iTV browsing. It was revealed that people's browsing decisions are determined by their transient moods. People's lifestyle is etched by mood directing decisions, shaping behaviour habits. When users are aware of the keywords in search, they prefer using the search engine. When they don't know the keyword, they browse by categories, drilling down for content until they find their desired results.

\subsection{Concerns and Controversies Theme Findings}

This study identified two types of user needs. Scenario $A$ type of users have no linguistic example to search by keyword, whereas scenario B type of users are simply driven by mood. The two types do not necessarily have to use the same browsing tool and user interface. Therefore, a new component, different to a mood-board, was designed to fit the purpose. A note to privacy was on automated technologies. Lastly, 'user needs' sub-theme advanced the study by redefining the purpose of mood-board style $\mathrm{UI}$ and by considering alternatives for different user needs.

\subsection{User Concepts Theme Findings}

User Concepts theme lead the design findings of the proposed interaction, its input and output mechanisms with regards to three related sub-themes. Namely, these were technologies, functions and design.

\subsubsection{Technologies}

The prevailing input technologies preferred were a blend of voice, body, gesture and image recognition, with a combination of automated and manual technologies to increase result accuracy.

\subsubsection{Functions}

A significant finding on functions sub-theme was the concept of mood tagging. A monitoring system, tagging the user's affective state, as felt before- duringand after watching a certain video. The user should find the mood tagging task as a worthy of their input effort and fun to use. Finally, the mood tagging system, could work in tandem, enriching the users experience in overall.

\subsubsection{Design}

The findings here suggest a three stage structure: the input stage, the desired output and the personalised results. For example, how users feel in the mo- ment, how they want to feel after watching a video and the video recommended to achieve the users goal. The results displayed in 'real-time', the recommendation section becomes a part of the users exploratory browsing experience, giving it a more playful tone.

Four new components emerged as a result of the conducted studies from the participants: a toggle button, to switch between personalised and social media results; an Instagram image uploading function; a plugin component for automated measurement technologies; and a Genre wheel of categories for serendipitous and tacit browsing. The Genre wheel was an innovative element. It emerged through the process of design iteration, to accommodate tacit forms of browsing, based on the fact that at least the Genre should be known, so that the user can drill further down into categories and themes to find the desired content.

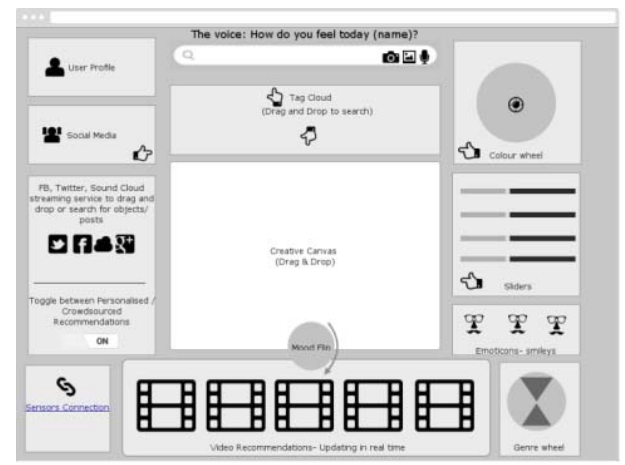

Figure 7: Prototype stage 1 - How do you feel today?

\subsection{RtD Model Findings}

RtD was selected due to three following reasons. First and foremost, it gave ground to the holistic approach that was underpinning this project, embracing both Affective Interaction theory and Design Practise. Secondly, it matched the scope of this study to sustain the users' behaviour and enrich it. Thirdly, it aims to leverage the knowledge in $\mathrm{HCl}$ community on designing for iTV user experience.

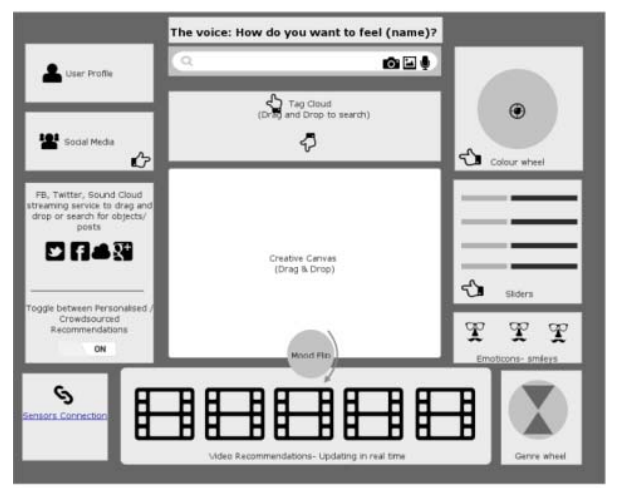

Figure 7: Prototype stage 2 - How would you like to feel? 
In alignment with the RtD process, the system has to be intuitive and effortless. The proposed system's invention is built on mood-based metadata parsed by a suggested new HTML 5 tag, <mood>happy $</$ mood $>$, so the user can browse and personalise iTV video content as such. Relevance is in the variety of different technologies employed. Lastly, the extensibility of the project relies on different technologies, functions and UI design used to search engine. A major understanding deriving from this research is a design agnostic stance towards the variety of UI interpretations, what matters is engaging the users.

\section{DISCUSSION AND FUTURE WORK}

This study advances Damasio's (2005) theory of emotion and reason combined, since it now becomes a tangible representation with the potential of future extension for further development by new technological functions and UI design advancements. What this study did not do, was to evaluate the designed prototypes. This iteration step is then suggested to develop the current study. Social iTV browsing was not considered, however users noted compromises they make on such occasions. Therefore, a study concentrating on this topic is encouraged.

\section{CONCLUSION}

This research set out to address the challenge of iTV content finding, to aid users' decision making process. It primarily explored the question: 'how could reason and emotion be combined to aid the user's decision-making process when browsing for iTV content'. Research findings revealed that people are driven by their temporary moods when deciding what iTV content to watch. However, the current state of iTV search engine technology lacks of affective mood support (Mosco 2004).

This study then investigated 'whether the user can browse for iTV content without the use of words'.

Observations on browsing patterns have shown that when the users are not aware of the keyword of the content they are looking for, then they navigate through categories towards the most relevant information they can find. In this research, this type browsing pattern was identified as 'tacit browsing'.

The third research question then was focused on 'designing an interaction (system /UI), that would support people's iTV browsing decisions'. As such, this research would embody the theory of emotion and cognition (Damasio 2005) with the study findings in practice. Based on users' preferences a search engine UI was designed to aid the users browsing decisions and enrich their iTV content find- ing experience. However, one could still argue that keywords must be used. In this case further research is recommended.

Finally this research focused on the cause of the user's browsing decisions underneath mood, that is, their motivation to be effortlessly engaged in a playful, yet effective way, to retrieve accurate and personalised recommendations. The role of interaction practitioners then is, to responsibly support people's needs and enrich their well-being, by seeding it with better experiences alike.

\section{REFERENCES}

Baird, B., Smallwood, J., Mrazek, M. D., Kam, J. W. Y., Franklin, M. S., and Schooler, J. W. (2012) Inspired by cistraction: mind wandering facilitates creative incubation. Psychological Science, 23(10).

Bechara, A., and Damasio, A. R. (2005) The somatic marker hypothesis: A neural theory of economic decision. Games and Economic Behavior, 52(2), 336372. doi:10.1016/j.geb.2004.06.010

Boehner, K., DePaula, R., Dourish, P., and Sengers, P. (2007) How emotion is made and measured. International Journal of Human-Computer Studies, 65(4), 275-291. doi:10.1016/j.ijhcs.2006.11.016

Bosma, W., Andr, E., and André, E. (2004) Exploiting emotions to disambiguate dialogue acts. In Proceedings of the 9th International Conference on Intelligent User Interfaces, pp. 85-92. New York, NY, USA: ACM. doi:10.1145/964442.964459

Braun, V., and Clarke, V. (2006) Using thematic analysis in psychology. Qualitative Research in Psychology, 3(2), 37-41.

Crabtree, A., Hemmings, T., Rodden, T., Cheverst, K., Clarke, K., Dewsbury, G., Hughes J., and Rouncefield, M. (2003) Designing with Care: Adapting Cultural Probes to Inform Design in Sensitive Settings. In Ergonomics Society of Australia, pp. 413. Retrieved from http://eprints.lancs.ac.uk/12354/

Dalen, O. (2011) Cut the crap: why deleting is improving. Retrieved September 1, 2013, from http://2011.csforum.eu/speakers/dalen

Eggink, J., Allen, P., and Bland, D. (2012) A pilot study for mood-based classification of TV programmes. In Proceedings of the 27th Annual ACM Symposium on Applied Computing, pp. 918-922. New York, NY, USA: ACM. doi:10.1145/2245276.2245455

Gao, Y., Bianch-Berthouze, N., and Meng, H. (2012) What does touch tell us about emotions in touchscreen-based. ACM Transactions on Computer-Human Interaction, 19(4), December. doi:10.1145/2395131.2395138 
Gaver, W. W., Boucher, A., Pennington, S., and Walker, B. (2004) Cultural probes and the value of uncertainty. Interactions - Funcology, 11(5), 53-56. doi:10.1145/1015530.1015555

Getty Images (n.d.) MoodstreamTM by Getty Images. Getty Images Inc. Retrieved December 6, 2013, from http://moodstream.gettyimages.com

Ghostly Discovery (n.d.) Retrieved April 2, 2014, from http://ghostly.com/discovery/play

Horizon. (2013) BBC Two - Horizon, 2012-2013, The Creative Brain: How Insight Works, How do our brains come up with ideas? UK: BBC TWO. Retrieved from

http://www.bbc.co.uk/programmes/p0160yfj

Li, J., Wang, Z., Wang, D., and Zhang, B. (2008) Interactive video retrieval with rich features and friendly interface. In Proceedings of the 2008 International Conference on Content-based Image and Video Retrieval, pp. 567-568. New York, NY, USA: ACM.

Lino, N. Q., Siebra, C., Araújo, J., Anabuki, D., Batista, M., Nóbrega, R., Amaro, M., and Lemos, G. (2011) Knowledge TV. In Proceedings of the 9th International Interactive Conference on Interactive Television (EurolTV '11), pp. 29-38. New York, NY, USA: ACM. doi:10.1145/2000119.2000126

Lucero, A., and Mattelmäki, T. (2011) Good to see you again: engaging users in design. In Proceedings of the 2011 Conference on Designing Pleasurable Products and Interfaces, pp. 11:1-11:8.

Mosco, V. (2004) The Digital Sublime. London and Cambridge: MIT Press.

Mural.ly - Visual Collaboration for Creative People (n.d.). Retrieved April 2, 2014, from https://mural.ly

Musicovery (n.d.). Retrieved April 2, 2014, from http://musicovery.com

Plutchik, R. (2001) The nature of emotions: human emotions have deep evolutionary roots, a fact that may explain their complexity and provide tools for clinical practice. American Scientist, 89(4), pp. 344350. Retrieved from http://www.jstor.org/stable/27857503

Rittel, H. J., and Webber, M. M. (1973) Dilemmas in a general theory of planning. Policy Sciences, 4(2), $155-169$

Ritter, S. M., Damian, R. I., Simonton, D. K., van Baaren, R. B., Strick, M., Derks, J., and Dijksterhuis,
A. (2012) Diversifying experiences enhance cognitive flexibility. Journal of Experimental Social Psychology, 48(4), 961-964.

Rogers, Y., Scaife, M., Harris, E., Phelps, T., Price, S., Smith, H., Muller, H., Randell, C., Moss, A., TayIor, I., Stanton, D., O'Malley, C., Corke, G., and Gabrielli, S. (2002) Things aren't what they seem to be: innovation through technology inspiration. In Proceedings of the Conference on Designing Interactive Systems Processes Practices Methods and Techniques (DIS '02), pp. 373-378. New York, NY, USA: ACM.

Sanders, E. B.-N. (2002) From user-centered to participatory design approaches. SonicRim. Design, pp. 1-8. Retrieved from

http://scholar.google.com/scholar?hl=en\&btnG=Sear ch\&q=intitle:From+User-

Centered+to+Participatory+Design+ Approaches\#0

Sciences, F., Martins, P., Chambel, T., and Langlois, T. (2011) MovieClouds: content-based overviews and exploratory browsing of movies. In Proceedings of the 15th International Academic MindTrek Conference: Envisioning Future Media Environments, pp. 133-140. Retrieved from http://dl.acm.org/citation.cfm?id=2181059

Stereormood srl (2008) Stereomood - turn your mood into music - free playlist for every mood in your life. Stereomood srl. Retrieved December 6, 2013, from http://www.stereomood.com

Vanderbilt, T. (n.d.) In-depth: the future of search, Wired UK. Retrieved September 1, 2013, from http://www.wired.co.uk/magazine/archive/2013/01/fe atures/the-future-of-search

yle.fi (n.d.). Retrieved April 2, 2014, from http://yle.fi

Zhang, S., Tian, Q., Huang, Q., Gao, W., and Li, S., (2009) Utilizing affective analysis for efficient movie browsing. In Proceedings of the 16th IEEE International Conference on Image Processing (ICIP), pp. 1853-1856. IEEE. doi:10.1109/ICIP.2009.5413590

Zimmerman, J., Forlizzi, J., and Evenson, S. (2007) Research through design as a method for interaction design research in $\mathrm{HCl}$. In Proceedings of the SIGCHI Conference on Human Factors in Computing Systems (CHI '07), 493-502. New York, NY, USA: ACM. doi:10.1145/1240624.1240704

Zimmerman, J., Kauapati, K., Buczak, A. L., Schaffer, D., Gutta, S., Martino, J., and Kurapati, K. (2004) TV personalization system. In Personalized Digital Television, Vol. 6, pp. 27-51. Springer Netherlands. 